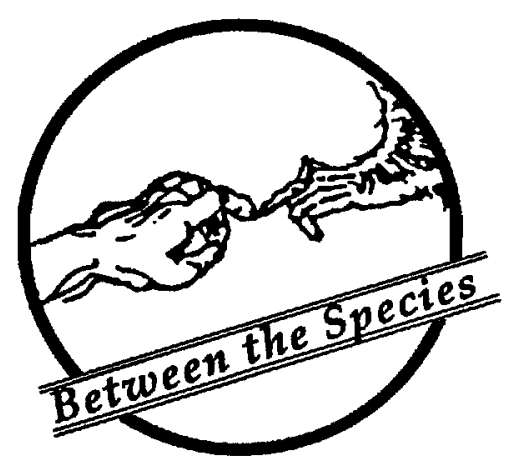

\title{
TO RECTIFY AN AMORAL DESIGN
}

\section{Maria Comninou \\ University of Michigan}

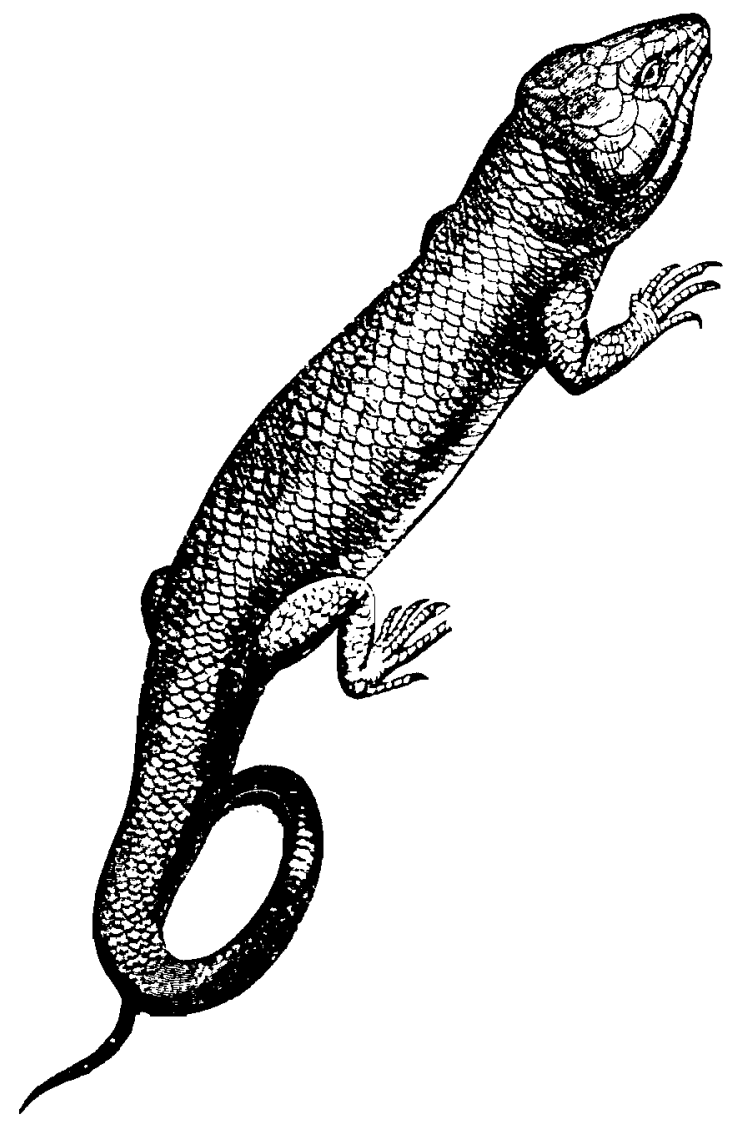

\section{The Original Design}

Theo was an undergraduate (male) in a major engineering school. In his senior year, he was given the project to design a product that met or exceeded the standards of current engineering practice. More specifically, the product ought to:

1. be efficient

2. be self-maintaining

3. be renewable and self-perpetuating

4. lead to increased productivity

Theo went over to the department of biotechnology, borrowed a segment of DNA, and with the help of a graduate student encoded on it all the desirable properties, as he interpreted them. He grafted the segment to a suitable microorganism, which he then sent in a minuscule spaceship to a distant planet. Theo and his project are long forgotten, but life still goes on, on planet earth.

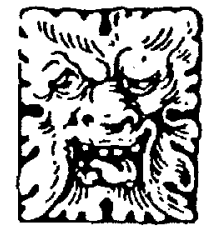

\section{COMMENT}


This story is not proposed as an alternative scenario of the origins of life on earth, not because it is implausible, but because it does not matter: the effects are as if the story were true. Regarding plausibility, the Nobel Prize laureate Francis Crick of DNA fame advocates the theory of directed panspermia. According to this theory, life on earth originated from microbes sent to earth aboard a spaceship from some higher civilization. Let us pretend, in what follows, that our story is correct and refer to Theo as a historical person.

Let us examine briefly how successful Theo's original design has been. Every living thing (plant or animal or in-between) strives to survive and procreate to the extent it finds possible. An animal lives mostly on other living things or their wastes. Herbivores eat or kill plants, carnivores kill animals: both types are predators, and possibly preys. A plant does not generally kill, but lives on soil nutrients, water and the sun's energy. Both plants and animals maintain, perpetuate and propagate themselves without additional input from Theo (efficiency and productivity). Since predators control prey population (plant or animal) and starvation controls them, there is a natural check on exponential growth arising from any single species.

Or is there? The human species has recently freed itself from any predators, and is now propagating at the expense of other living things who are becoming extinct. The human race has become as clever as Theo and is foiling his plan. Unless, of course, Theo has already put some conditional statements in his genetic code, which at the appropriate moment will cause the extinction of the dominant species: he has programmed the downfall of the dinosaurs, and has a similar fate in store for humans.

\section{The Necessity of Killing}

The only living things that do not kill for food are the plants (with a few exceptions of carnivorous plants). Plants are the only humane beings on the planet, the epitome of a preservationist, frugal design, in harmony with the resources of the earth and the elements. It is the goal we, moral humans, should strive to achieve.

Was killing part of Theo's design? It is doubtful. Like modern engineers, he had good intentions but failed to foresee the full chain of implications embedded in the tenets of his design. Killing was a manifestation of a code of optimization which operated without any ethical constraints. In other words, it was more costeffective for a species to feed on another, than to put down roots and turn toward the sun. Animals were an aberration born out of maximum efficiency and self-promotion. In their turn, plants, like rabbits, try to procreate excessively, because they have so many predators. The end result is continuous struggle between the forces of expansion and the forces of control. In this struggle, and at least after the last recorded extinction, there were no clear winners until technology tipped the balance.

The law against killing marks the revolt of the moral man (I doubt whether women were ever asked, even in those early days) against an inhumane design and his attempt to rectify it.

\section{The Invisible Line}

The conflict created by the no-kill commandment was resolved by "drawing the line." The line is conveniently drawn as circular, with the individual at its center. Enlightened individuals argue about the precise boundary: does it include cockroaches and mosquitos? does it include vegetables? bacteria? Recent advances in behavioral microbiology, says Harold Morowith in Mayonnaise and the Origin of Life, imply that single-cell organisms are beings that can sense and that are capable of purposeful activity. Attempts are also made to draw the line on the basis of some important, for humans, feature: pain, anticipation, thought, type of offspring, etc. Such attempts are futile; not only are we at a loss to tell who feels or thinks what without direct extrapolation from our own experience, but also our measures are only for humans. Other species have not given us their consensus of what is important for them. But even if we grant that thought is, for example, the criterion, who is to determine whether dandelions think or not? We can for sure conclude that they do not think like us by using the scientific method, in which man is the yardstick. However, "thought" does not have to be something associated with brain waves, and comparisons are only good if we are trying to determine clones of ourselves.

The fact is that the necessity of killing has precluded the drawing of the line. If ever drawn, the 
line has remained invisible. It will be an event of enormous significance when the line is at last drawn, if only to include the human race alone. For what is the meaning of having a forbidding boundary when it is casually crossed by individuals, and purposefully broken by warring nations?

\section{Ecology and the Bad Dog}

Ecology, including deep ecology, is a classic case of correct diagnosis and bad therapy. It is dominated by the zoo mentality: collect the last specimens and preserve them, preserve viable populations, preserve patches of habitats, etc. Like zoo keepers, ecologists judge species by subjective and popularity standards, and single them out for preservation. Sincere ecology is incompatible with the human race of the technological age, and if one species had to be sacrificed for the good of the rest, that ought to be the human species. If one species ought to be controlled by culling, that ought to be the human species. Ecology ought to go hand in hand with human birth control, veganism if not fruitarianism, and egalitarian distribution of resources. Otherwise, it is simply a self-righteous theory.

\section{Animal Rights: No Bad Dog}

Despite claims to the contrary, animal rights is still an inconsistent and impractical theory. It is inconsistent for the following reasons: According to the theory the claims of the individual animal supercede those of a species. It is the individual animal who lives, feels and pursues the goals of its existence. The species is simply a convenient idea, the shadow of reality. If this statement is true, there is no way a hierarchy can be imposed on animals, and the line cannot be drawn anywhere. There is nobody to decide to whom the bill of rights applies. Each "species" is simply a congregation of individuals to be judged and treated as single entities. This elephant has as much right to exist undisturbed by human interference as that pregnant female bloodsucking mosquito (male mosquitos are vegetarians). We are already in a fine tangle. The human race, which is prepared to relinquish its superiority and special status on the altar of animal rights, is the one which must assume the role of policing, judging, and managing, without having received any other animal's tacit or otherwise endorsement.

If we cannot dismiss animals, we cannot dismiss bacteria, and we cannot dismiss plants. Science has already blurred the line between animal and vegetable life, and in any case, any distinction it may provide would be just as arbitrary as classifying birds by type of feather. Physiological differences do not constitute a difference in the eye of moral justice. As it is impossible to refrain from consuming and to protect all living beings, animal or otherwise, animal rights by itself as a theory to guide behavior is impractical.

Animal rights theorists and activists are impatient and tend to dismiss those who say "how about cockroaches?" or "how about plants?". This is because they consciously or unconsciously feel they are at one end of a long tunnel, and it is premature or counterproductive to ask what is at the other end. However, it is important to know the guiding principles along the way, even though the end is not remotely foreseeable. In human affairs, there are those who argue in favor of "first things first," and historically this is indeed how rights have been extended. The decision on what or who is to be first does not even depend on the strength of numbers. It usually rests on the prejudices of those controlling the forces of change. For example, the issue of women's rights was typically dismissed by socialist and communist revolutionaries up to the present as secondary. The same is true of most modern socalled "freedom fighters" or "terrorists" of either side of the political fence. If animal rights is the logical culmination of human rights, and the epitome of humanitarianism, it cannot afford to dismiss incongruities on the grounds of ignorance. Ignorance about the boundaries of individuality of life in a species should count in favor, and not against the inclusion of the species in the bill of rights as is typically argued by Regan.

The animal rights movement is the movement with the most radical and far-reaching implications ever seriously considered. It is unrealistic to expect the present-day philosophers to formulate an animal rights theory which is consistent and practical. On the contrary, it seems that the work of philosophy can never be completed in a non-static and less-thanideal world: as soon as a theory is invented to resolve the latest moral dilemma, another dilemma surfaces just outside it. 